\title{
USAGE OF 3-D BASED METHODS FOR THE DETECTION OF AGGREGATE MICROTEXTURE
}

Some of the available advanced three-dimensional based methods for measurement of microtexture have been described. These methods are generally divided into three categories, namely image analysis based methods, laser based methods and photogrammetry methods. Moreover, a particle of aggregate can be captured using a stereomicroscope and using the appropriate software to obtain a 3-D image of investigated aggregate. Software also includes an interactive measurement tool that allows to obtain a profile of aggregate particle. The stereomicroscope method is also presented in the paper. The usage of the microscope for the detection of aggregate microtexture allows to eliminate some of the disadvantages of described methods and provides additional opportunities for microtexture evaluation.

Keywords: Microtexture, aggregate, 3-D based methods, microscope method.

\section{Introduction}

Microtexture is defined as configuration of particular peaks on the surface of aggregate particles. Microtexture is very considerable parameter in term of skid resistance and mainly influence friction at low speeds on dry pavement surface [1]. Microtexture is also necessary to assure high friction value between a tire and pavement [2]. Microtexture is characterized by wavelengths and amplitudes in the range between $1 \mu \mathrm{m}-0.5$ $\mathrm{mm}$ [3].

In [4], the microtexture is defined as the angularity of the aggregate particle, which represents geometric attribute of the aggregate to have sharp edges (Fig. 1). The more shaped surface of the aggregate, firmer and also sharper material of surface means that better and more lasting friction is expected.

It is clear that the possibilities of measuring microtexture and the knowledge of the roughness of aggregate particles are particularly important for road traffic safety.

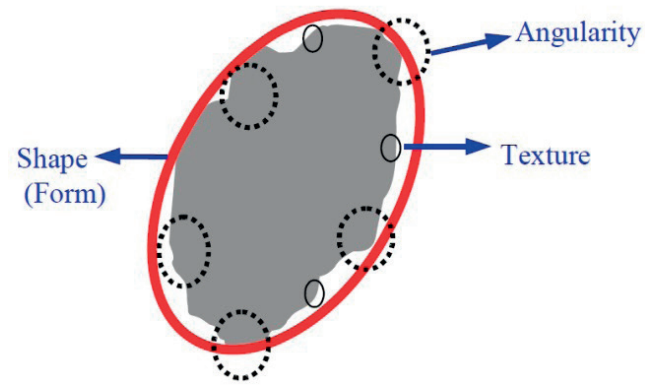

Fig. 1 Geometry of aggregate [5]
Variety of methods has been developed for detection of microtexture. These methods can be generally divided into manual measurements, detection of microtexture based on comparison and digital image analysis methods. The manual methods and the methods on basis of comparison are easy to realize, but are considered as subjective and time consuming. The current development of technique provides enhancement of microtexture detection methods by the methods obtaining outputs in the form of three-dimensional image of investigated particle.

\section{Methods based on an image analysis}

Determination of microtexture using digital image analysis methods (DIAM methods) generally consists of image acquisition, the image processing (image editing) and subsequent evaluation of the image by various methods. The image analysis means conversion of image to data. Because the digital image is used, all transformations and calculations are implemented in pixels. In most cases, the evaluation is carried out by various mathematical algorithms in different computing programs. Many of used algorithms are complicated and very difficult to programming. Therefore, this method requires considerable computer knowledge and longer processing time.

A wide range of complex systems based on digital image analysis which allow obtaining a $3 \mathrm{D}$ view of the particle and then evaluation of aggregate microtexture have been developed. These systems generally consist of mechanisms for obtaining high-quality images of aggregates and evaluation software. These

\footnotetext{
* Zuzana Florkova

Department of Highway Engineering, Faculty of Civil Engineering, University of Zilina, Slovakia

E-mail: zuzana.florkova@fstav.uniza.sk
} 
systems include, for example, the system UIAIA (University of Illinois Aggregate Image Analyzer - Fig. 2).

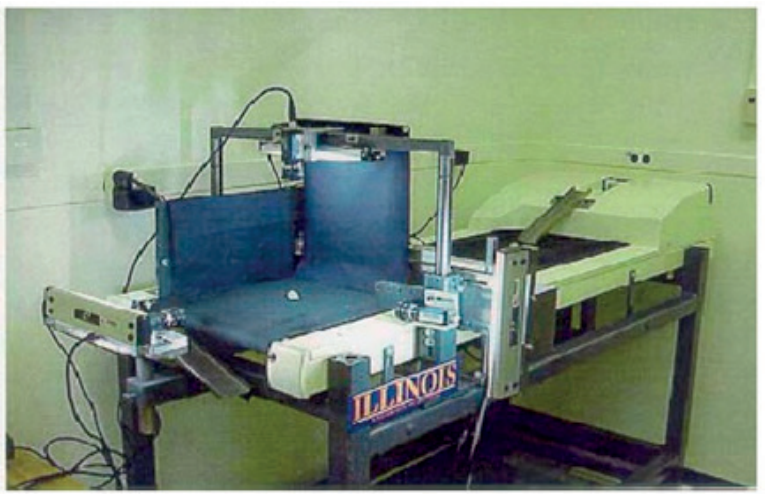

Fig. 2 UIAIA system [5]

The UIAIA uses three cameras to capture projections of a particle from three orthogonal directions. The UIAIA features a moving conveyor belt that carries the individual aggregate particle into the view of a sensor, which detects the particle and immediately triggers the cameras. The use of three images for each particle allows creating the 3-D view of each particle. This system uses for microtexture evaluation the outline slope angularity index. The outline slope angularity index measures the angularity of a particle as change in the slope of the particle polygon outline (Fig. 3).

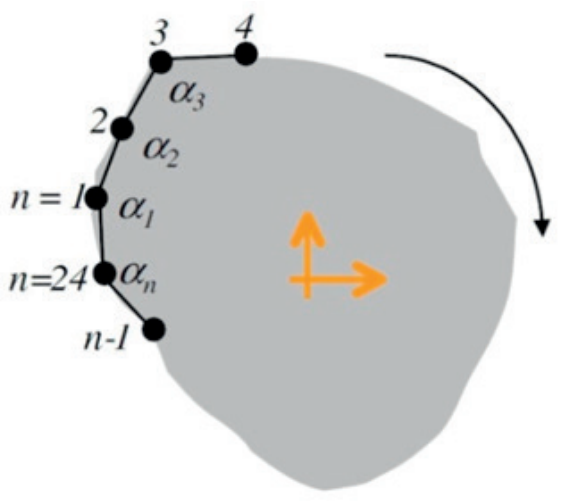

Fig. 3 The polygon outline of particle [6]

The main disadvantage of these systems is that the available parameters for angularity characteristics of aggregate particles by these methods are based on analysis of 2-D images. Despite of this disadvantage complex systems provide outputs in the form of 3-D view of the particle, the appropriate evaluation software of these systems allows microtexture evaluation of individual particles only by 2-D parameters. Then, the resultant value is assessed as the average value of the parameters obtained from the evaluation of 2-D images of many projected sides of an aggregate particle.

In general, disadvantage of 2-D parameters based on digital image analysis is that these parameters allow to capture only the larger peaks on the surface of aggregate particles. As the result of this, only the upper part of microtexture range is included into the evaluation. Used microtexture parameters are described in the [6].

\section{3D laser scanning device}

This laser-based method is intended for simple threedimensional (3-D) reconstruction of aggregate particles (Fig. 5). The 3-D laser scanning device in Fig. 4 uses advanced noncontact laser sensors to scan an object in three dimensions. Aggregate particles are scanned on rotating plate using a laser beam, which travels vertically up the rotating object to generate three dimensional digital scan file. Data processing software is an integral part of the 3-D laser device [7].

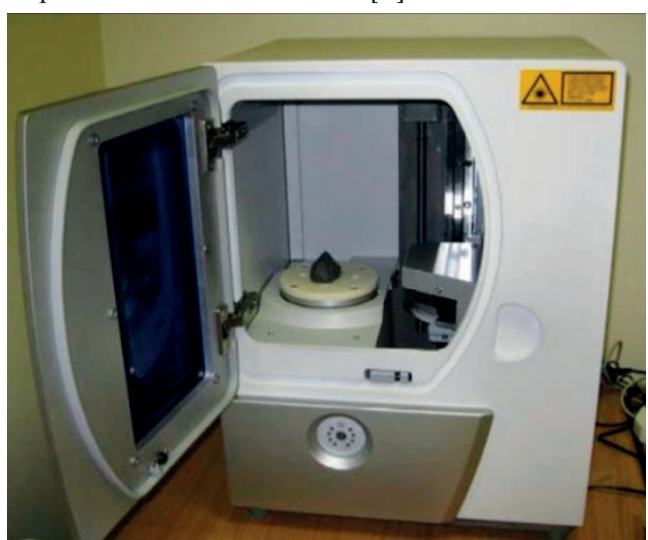

Fig. 4 3D laser scanning device [8]

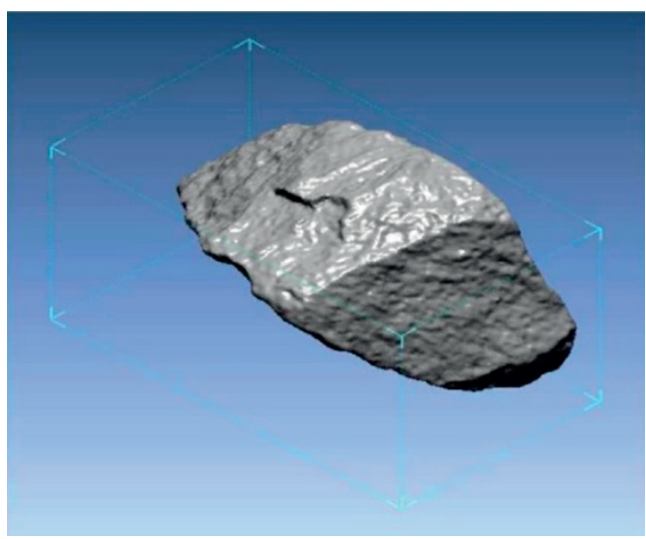

Fig. 5 3D image of aggregate particle obtained by 3D laser scanning device [8]

This method has the same disadvantage as in the case of the above presented methods of digital image analysis. The entire evaluation is carried out automatically, but by the 2D parameters. The capture of the entire field of microtexture values is also 
problematic, because of the laser device software, which scans objects with a $0.1 \mathrm{~mm}$ scanning resolution.

\section{Photogrammetry method}

The photogrammetry method is another method for the detection of microtexture, which allows obtaining the outputs in the 3-D form (Fig. 7). Photogrammetry is a set of methods for obtaining the coordinates of prepared aggregate sample (Fig. 6) from analogue or digital photography. The principle of this method is in obtaining photographs, getting the coordinates of the surface using the appropriate software and the further transformation of the coordinates in the 3-D model.

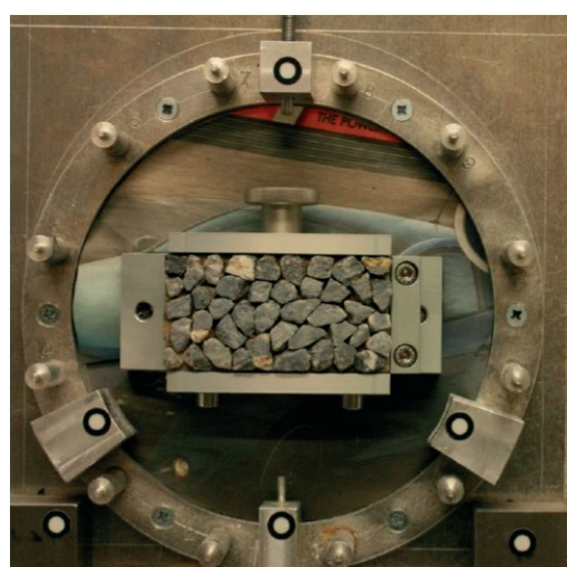

Fig. 6 Sample of aggregates used for obtaining the $3 D$ aggregate image by photogrammetry method [9]

This method uses software Mountain Map for microtexture evaluation. The advantage of this software is that it enables to include into evaluation the entire particle surface by volume characteristics. One of the possibilities is to use the material ratio curve so-called the Abbott-Firestone curve.

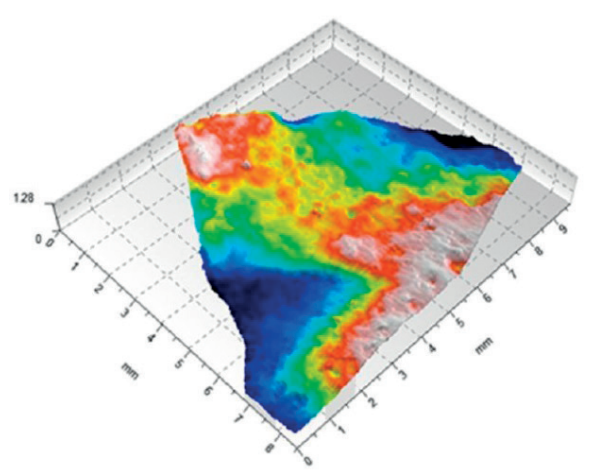

Fig. 7 The photogrammetry method used for obtaining the 3D aggregate image [9]
Using the Abbott curve, a graphical analysis can be performed in order to retrieve volume parameters characterizing the roughness of aggregate particle. According to Fig. 8, the curve distributes the surface texture into four volume parameters: $\mathrm{Vmp}$ (peak material volume), Vmc (core material volume), Vvc (core void volume) and Vvv (valley of the void volume) [9]. Then, the resultant characteristic of microtexture represents the volume parameter Vmp (peak material volume) (Fig. 9). The downside is that this method requires considerable computer knowledge and longer processing time.

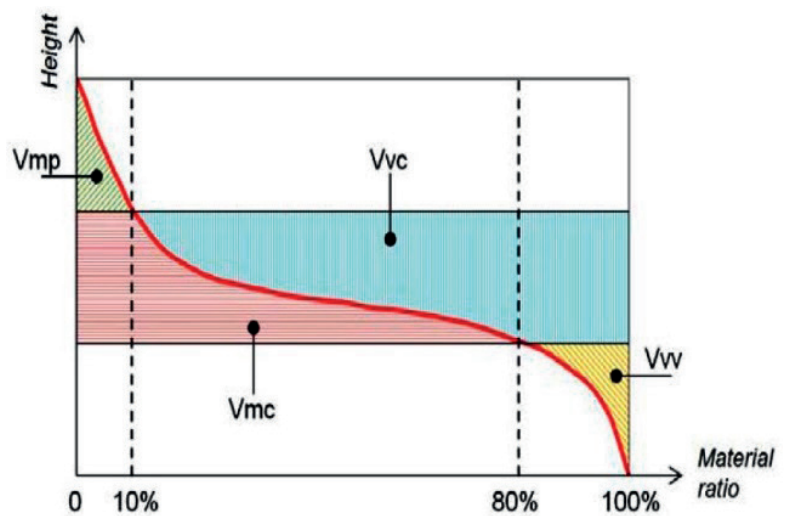

Fig. 8 Principle of Abbott-Firestone [10]

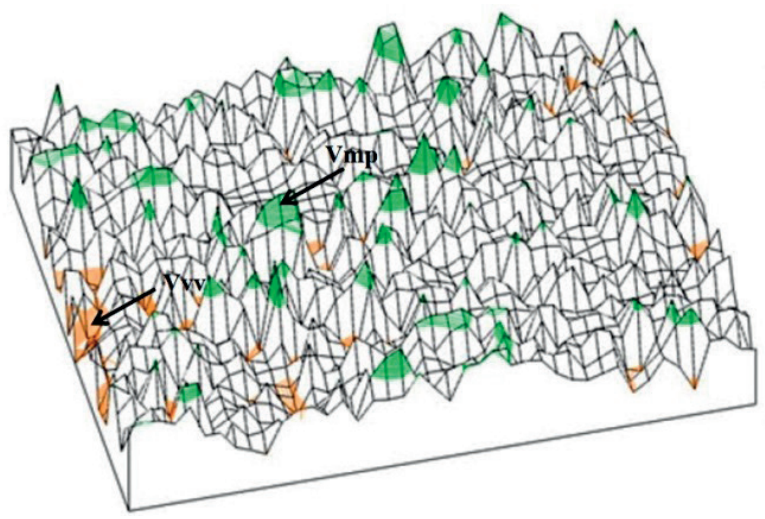

Fig. 9 Characteristic of microtexture represented

by the volume parameter Vmp (peak material volume) [10]

\section{Usage of microscope method for the detection of microtexture}

It is possible to scan a particle of aggregate by a microscope (e.g. NIKON AZ 100). On the basis of the software Nis Elements $\mathrm{D}$ it is possible to achieve a 3-D view of investigated aggregate (Fig. 10). This view can be exported into the wrml (Virtual Reality Modelling Language) format. It makes possible to image the aggregate in 3-D also apart from the software Nis Elements D in the workspaceof different programs (e.g. in the workspace of 
Matlab program - Fig. 11). The appropriate software also includes an interactive measurement toll EDF profile. Usage of this one allows obtaining the coordinates of singular profiles of aggregate. The acquired profile of aggregate is then expressed by $\mathrm{x}(\mathrm{y})$ and $\mathrm{z}$ coordinates. This data file can be also exported to various formats.

The principle of 3-D view acquirement by microscope rests in scanning of aggregate particle by cameras at different height levels. Aggregate particle is scanned on the microscope stage, which is vertically moved in micro steps by rotary knob. Then, the software creates the resultant 3-D image from the images obtained by scanning. The accuracy of the obtained output depends on the before selected range of scanning, of the number of steps and the required zoom level.

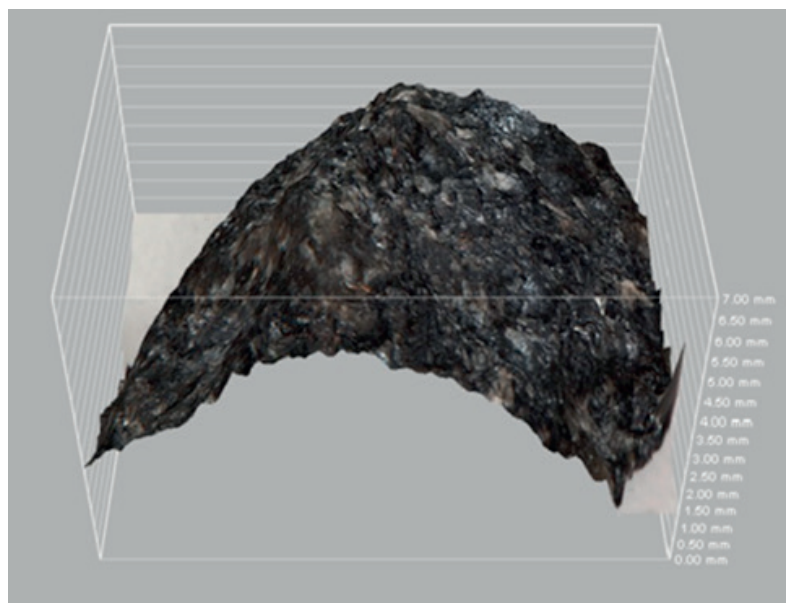

Fig. 10 Three-dimensional view of aggregate particle obtained by microscope

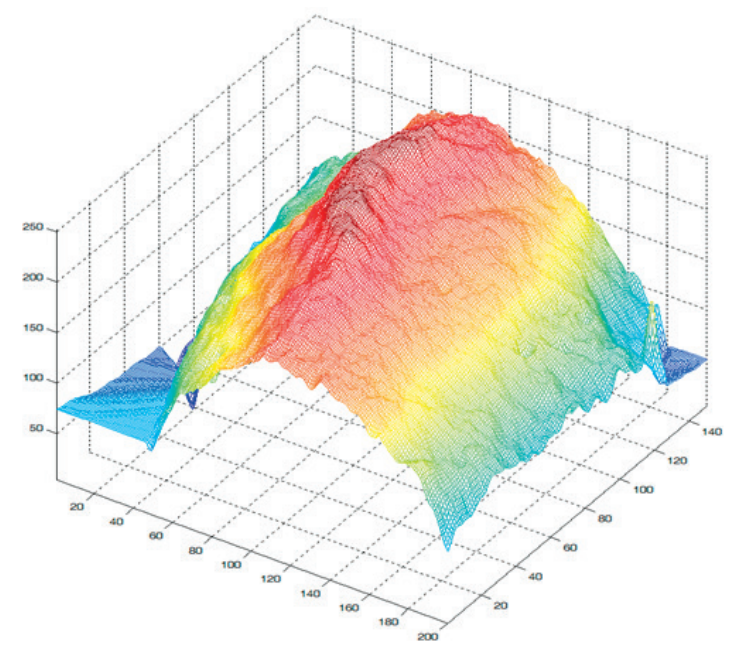

Fig. 11 Image of aggregate in the Matlab program workspace

The main advantage of this method is the possibility to image aggregate particle in 3-D apart from the software Nis Elements D in the workspace of different programs. Then, a program can be used for following evaluation. The program allows microtexture evaluation by 3-D parameters and eventually deals with creation of new parameters, which provide new possibilities of microtexture evaluation using the surface areas and volumes.

An aggregate particle can be captured by microscope in different zoom levels. It provides the capturing of the entire field of microtexture values and it gives more accuracy and better representativeness of results (compared to other detection methods of microtexture).

In all, the process of measuring by microscope is not timeconsuming and it is quite simple.

\section{Conclusion}

The possibility that microtexture information can be obtained from three-dimensional data by the 3-D image methods involves the series of advantages in monitoring the aggregate microtexture. By these outputs, it is possible to detect aggregate microtexture in a more realistic way. In particular, it allows determination of microtexture characteristics with high accuracy and reliability of the results when compared to other used microtexture detection methods.

Despite of these advantages, these methods have still some limitations. The main limitation of these systems is that the systems provide outputs in the form of a 3-D view of the particle and the appropriate evaluation software of these systems allows microtexture evaluation of individual particles only by 2-D parameters.

Generally, the approach to quantification of aggregate microtexture based on the microscope methods is not commonly used as compared to other 3-D based methods. The usage of the microscope method for the detection of aggregate microtexture allows eliminating some of the disadvantages of described methods and mainly the limitation in the form of resultant 2-D image analysis.

Using these outputs in the tree-dimensional form obtained by microscope method gives possibility to include into the evaluation the entire surface of the investigation particle of the aggregate and also deals with microtexture evaluation based on volume characteristics.

The previous considerations can be used as a starting point for further studies of aggregate microtexture by the microscope method. Working with a 3-D surface, it is possible to go one step further and move to evolution of new parameters, with the main aim to find the most suitable parameter as the best characterization of microtexture. Possible further applications of these indicators can be very useful for more complex evaluation of pavement microtexture. 


\section{Acknowledgement}

The paper is an output of the project VEGA 1/0804/12 Influence of material composition of asphalt on characteristics of pavement surface texture, noise emission and air pollution supported by funds of Scientific Grant Agency of the Ministry of Education, science, research and sport of the Slovak Republic.

\section{References}

[1] HIBBS, B., LARSON, R.: Tire Pavement Noise and Safety Performance: PCC Surface texture Technical Working Group. Report No. FHWA-SA-96-068. Federal Highway administration. Washington, D.C., May 1996 [cit. 2015-29-03]. Available on: http://isddc.dot. gov/OLPFiles/FHWA/013169.pdf

[2] KOVAC, M. et al.: Diagnostics of Serviceability Parameters of Pavements, EDIS: University of Zilina, 2012, ISBN 978-80-554-0568-1.

[3] STN EN ISO 13473-5. Characterization of Pavement Texture by Use of Surface Profiles. Part 5: Determination of Megatexture, ISO 13473-5:2009

[4] KIM, Y., SOUZA, L.: Effects of Aggregate Angularity on Mix Design Characteristics and Pavement Performance. December 2009 [cit. 2014-08-05]. Available on: http://neltap.unl.edu/Documents/NDOR/Aggregate_Angularity_on\% 20Mix_Design.pdf

[5] MASAD, E. et al.: Appendixes to NCHRP Report 555: Test Methods for Characterizing Aggregate Shape, Texture, and Angularity. May 2005 [cit. 2014-25-04]. Available on: http://onlinepubs.trb.org/onlinepubs/nchrp/nchrp_w80.pdf

[6] TAFESSE, S. et al.: A New Image Analysis Technique to Quantify Particle Angularity. [cit.2014-30-04]. Available on: http://kth. divaportal.org/smash/get/diva2:499535/FULLTE XT01

[7] KOMBA, J.: Analytical and Laser Scanning Techniques to Determine Shape of Aggregates Used in Pavements. June3. [cit. 2015-13-03]. Available on: https://www.google.sk/search?client=opera\&q=Analytical+and+laser+scanninf+technuques+to+dtermine + shape + properties + of + aggregates + used $+i n+$ pavements\&sourceid $=$ opera\&ie $=U T F-8 \& o e=U T F-8 \&$ gws_rd $=$ cr\&ei $=$ xW4SVY71DcXyUvvYggL

[8] ANOCHIE-BOATENG, J. et al.: Evaluation of 3D Laser Device for Characterizing Shape and Surface Properties of Aggregates Used in Pavements. [cit. 2015-13-03] Available on: https://www.google.sk/search?client=opera\&q=+Evaluation+of $+3 \mathrm{D}+$ laser + device + for + characterizing + shape + and + surface + properties + of + aggregates + used + in + pavements $\&$ sourceid $=$ opera\&ie $=U T F 8 \& o e=$ UTF8\&gws_rd=cr\&ei=_3ASVaTFNsbfUdWcgsgF\#q=3d+laser+scanning + device+aggregate

[9] MCQUAID, G. et al.: Use of Close Range Photogrammetry to Assess the Micro-texture of Asphalt Surfacing Aggregate. Intern. J. of Pavements Conference, Sao Paulo, 2013.

[10] BITELLI, G. et al.: Laser Scanning on Road Pavements: A New Approach for Characterizing Surface Texture. Sensors, July 2012, ISSN 1424-8220. 